\title{
Imaging Findings in Late-Onset Rectal and Vesical Bleed in Patients Who Have Undergone Radiation Therapy for Pelvic Neoplasms
}

\author{
Jagadeesh Pasupuleti ${ }^{\oplus 1}$, Yugandhar Samireddypalle ${ }^{\circledR 2}$, Guntaka Srujana ${ }^{\oplus 1}$, Ramavath Ravi Naik ${ }^{\oplus 1}$, Sabarinath Eada ${ }^{\circledR 1}$, K.S. Vedaraju ${ }^{\circledR 3}$ \\ ${ }^{1}$ Postgraduate, Department of Radiology, Narayana medical college, Nellore, Andhra Pradesh, India, ${ }^{2}$ Senior resident, Interventional Radiologist, Department of \\ Radiology, Narayana medical college, Nellore, Andhra Pradesh, India, ${ }^{3}$ Professor \& Head, Department of Radiology, Narayana medical college, Nellore, Andhra \\ Pradesh, India.
}

\section{Abstract}

Radiation-induced vessel injury is described more than a century ago and remains a persistent clinical problem, despite advances in the field of radiation oncology. Treatment of pelvic neoplasms with radiotherapy may result in chronic radiation toxicity, especially Haemorrhagic radiation cystitis and chronic radiation proctitis. We discussed the computed tomography (CT) findings of three cases with a history of radiotherapy to the pelvis presenting with hematuria and hematochezia. Contrast-enhanced CT could diagnose the cause and site of bleed. All these patients were managed successfully by endoscopic coagulation.

Keywords: Computed Tomography, Imaging Findings, Per Rectal Bleed, Hematuria.

Corresponding Author: Yugandhar Samireddypalle, Senior resident, Interventional Radiologist, Department of Radiology, Narayana medical college, Nellore, Andhra Pradesh, India.

E-mail: yugu.samireddypalle@gmail.com

Received: 30 September 2020

\section{Introduction}

Radiation therapy is one of the main treatment modalities for pelvic neoplasms. New modifications and advancements in radiation therapy like external beam and brachytherapy have reduced malignancy recurrence. ${ }^{[1,2]}$ With an improvement in morbidity and mortality, there is an increasing encounter with late radiation toxicities. Ischemia, fibrosis, and mucosal bleeding due to abnormal leaky telangiectatic vessels are the usual late effects of radiation in the bowel and urinary bladder. ${ }^{[3-5]}$ While mild degrees of hematuria and hematochezia can be managed medically, severe bleeding usually requires endoscopic or endovascular interventions. ${ }^{[6]}$ We now discuss three cases with a prior history of radiation to the pelvis a few years back, presenting with hematuria and bleeding per rectum. We discuss the CT angiogram imaging findings in each case.

\section{Case 1}

A 72-year-old female patient who had undergone radiotherapy 11 yrs back for carcinoma cervix came with complaints of moderate to severe per rectal bleed for three days and was admitted to our hospital for further management. She was clinically stable at the time of admission and was passing frank blood per rectum. The patient underwent a $\mathrm{CT}$ angiogram of the abdomen and pelvis, which revealed long segment mural thickening involving the entire rectum. There is diffuse fat stranding in the mesorectum with mild thickening of the mesorectal fascia, suggesting chronic radiation proctitis [Figure 1,2]. There is active extravasation of contrast material from the middle rectal artery into the rectum [Figure 2]. There was also a rectouterine fistula with large communication of the rectal contents into the vagina [Figure 3]. The patient was managed successfully by endoscopic thermal coagulation.

\section{Case 2}

$65 \mathrm{yr}$ old female came to the emergency department with complaints of hematuria for 3 days. She was clinically stable and had a history of pelvic irradiation 30 yrs back for carcinoma cervix. The gross examination of the urine was dark red. The patient underwent a CT urogram, which revealed a hyperdense mass in the bladder's dependent portion showing no enhancement on post-contrast images [Figure 4]. This hyperdense mass was moving to a dependent portion on change in position, suggestive of hematoma [Figure 5]. No Active extravasation of contrast was noted in the arterial phase of the angiogram. 
She underwent cystoscopy, which revealed small bleeders from the posterior wall of the bladder. All the bleeders were successfully coagulated.

\section{Case 3}

72 yr old female with a history of carcinoma cervix underwent radiotherapy $20 \mathrm{yrs}$ back now came with complaints of gross hematuria for one day. On examination, she was clinically stable except for tachycardia and was passing frank blood in the urine. The patient underwent a plain CT angiogram of the abdomen and pelvis, which revealed a large hyperdense hematoma within the lumen of the urinary bladder and active extravasation of contrast from the left posterolateral wall of the bladder [Figure 6,7]. She also underwent successful cystoscopic thermal ablation on the same day of admission.

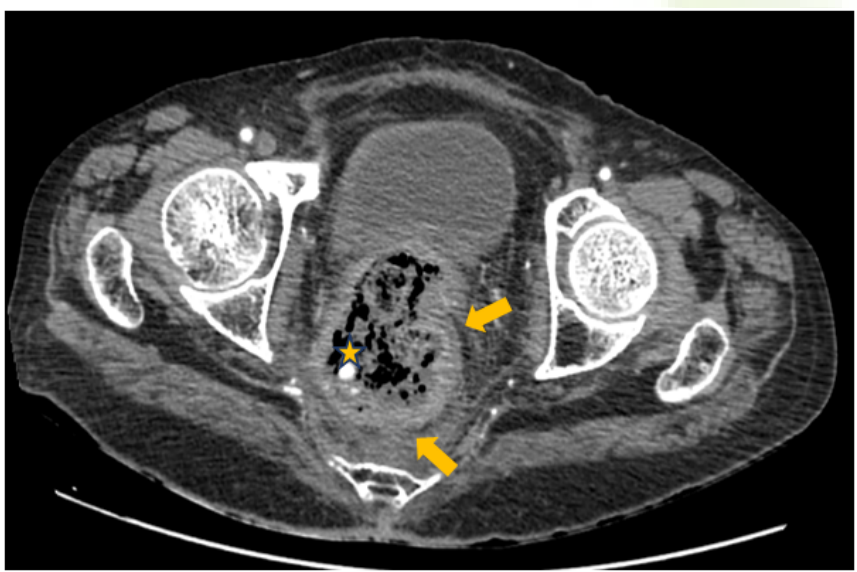

Figure 1: Axial section of CT angiogram pelvis, arterial phase, demonstrating diffuse circumferential mural thickening of the rectum with fat stranding in the mesorectum, suggesting changes of chronic radiation proctitis (arrows). The note is made of active extravasation of contrast from the right lateral wall of the rectum (asterisk)

\section{Discussion}

Radiation complications of the pelvis is a well-recognized entity. In the large intestine, like the rectum, early radiation injury changes cause damage to the epithelium and result in inflammatory changes. Late changes can be seen as early as six months and include fibrosis and endarteritis, resulting in ischemia. More severe ischemia will lead to ulceration, perforation, and fistula formation. ${ }^{[3]}$

The pathological study revealed increased intima thickness with increased proteoglycan content, which is quite different

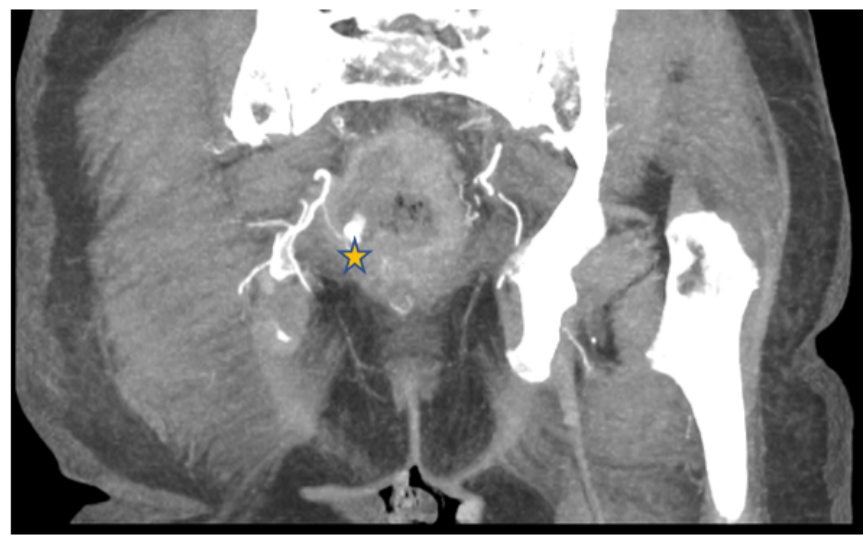

Figure 2: Coronal reformatted MIP (maximum intensity projection) image demonstrates an active contrast leak from one of the middle rectal artery (asterisk).

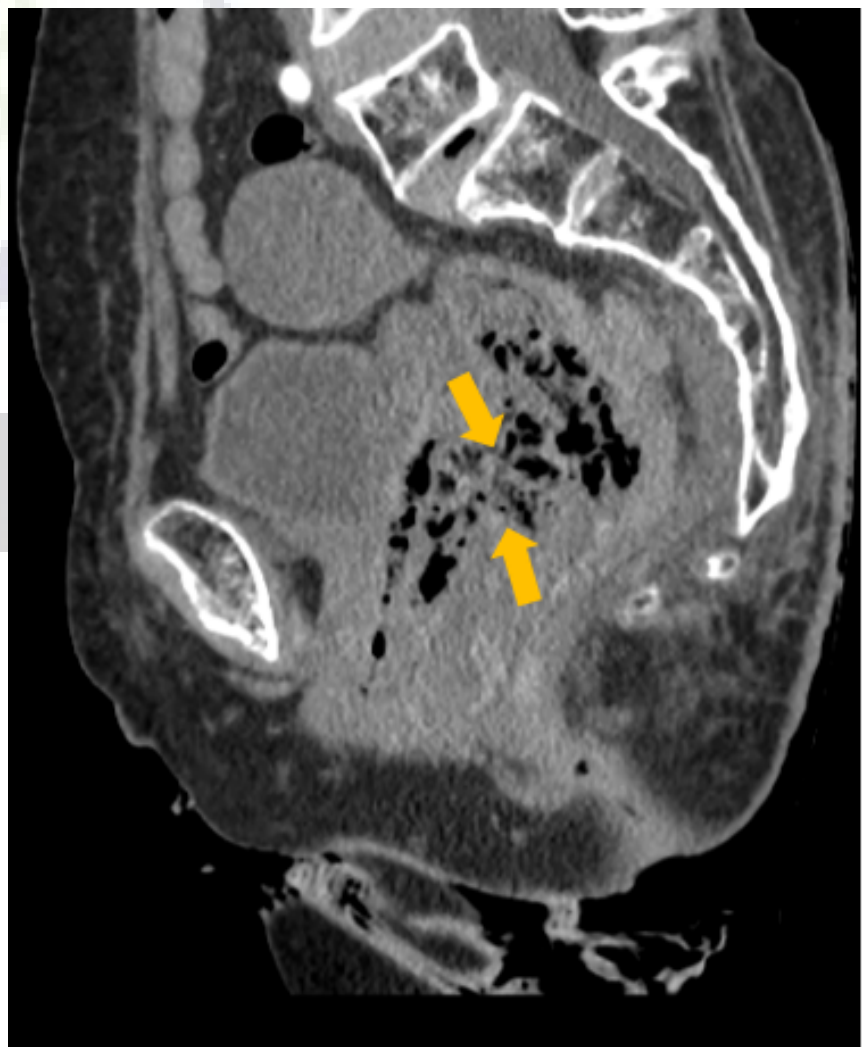

Figure 3: Sagittal reformatted plain CT pelvis demonstrating breach in the opposing walls of rectum and cervix with feculent material in the vagina, suggesting rectouterine fistula. (arrows) 


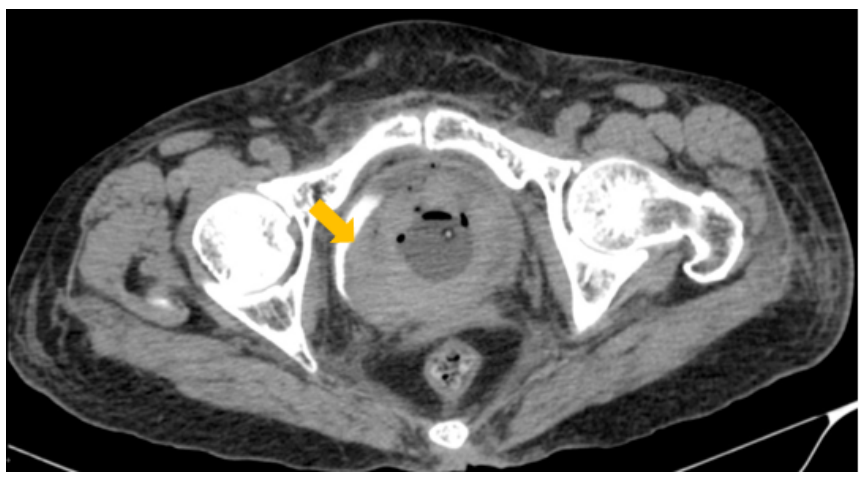

Figure 4: Axial CT scan of pelvis delayed phase demonstrating large hyperdense hematoma within the urinary bladder(arrow). A note is made of a foley bulb in situ and excreted urine in the bladder, opacified by contrast in the delayed phase.

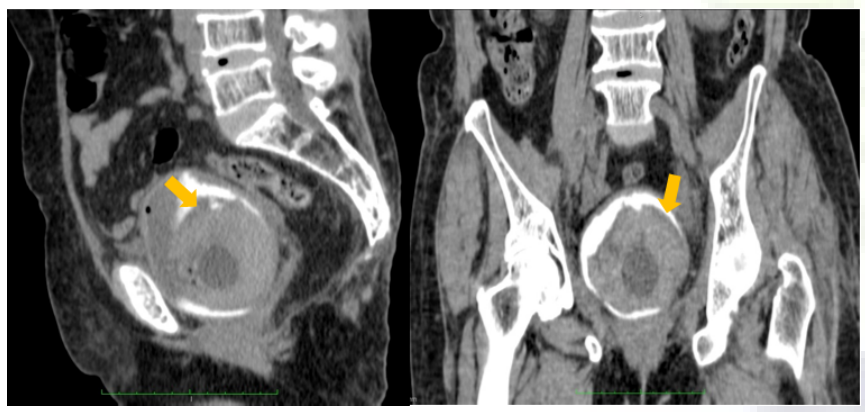

Figure 5: Reformatted sagittal and coronal images of pelvis demonstrating large hyperdense bladder hematoma (arrow).

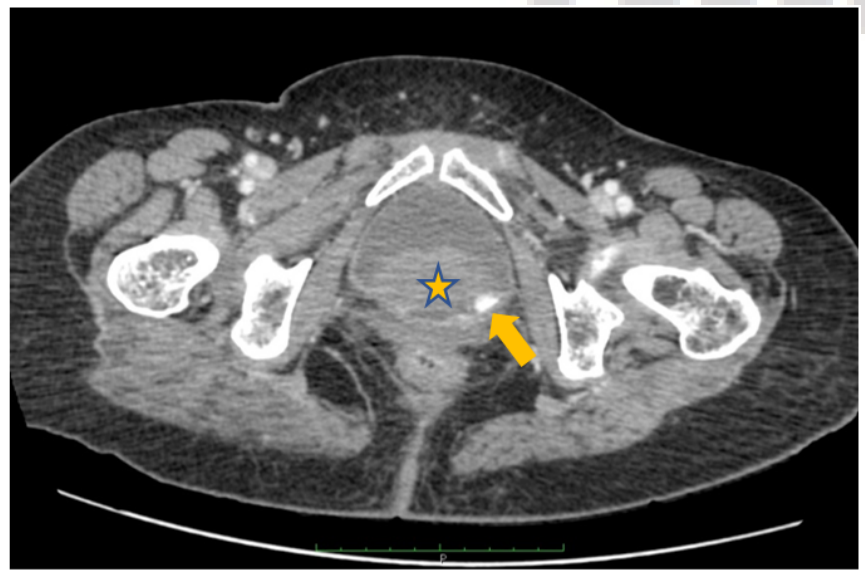

Figure 6: Axial CT angiogram of pelvis demonstrating hyper dense bladder hematoma (asterisk). There is active extravasation of contrast from the left posterolateral wall of the bladder (arrow)

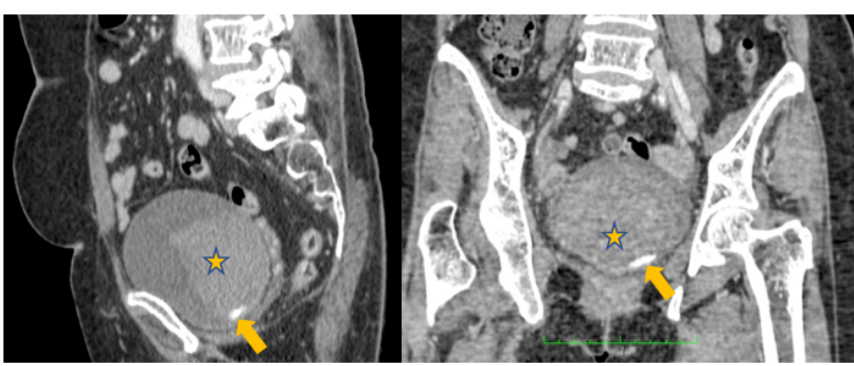

Figure 7: Reformatted sagittal and coronal pelvis images demonstrate large hyperdense bladder hematoma (asterisk) with active extravasation of contrast (arrow).

from age-related atherosclerosis. ${ }^{[2]}$

The mucosal vessels compensate for this ischemia and develop telangiectasia, which is the cause of bleeding. [3]

There is an increased risk of developing symptomatic radiation enteritis in patients with an underlying vascular occlusive disease like hypertension, diabetes, and cardiovascular disease. Endoscopy is always mandatory in all cases of rectal symptoms with prior history of radiation to rule out malignancy. In proctoscopy, Chronic findings of severe vascular changes such as capillary telangiectasia, the formation of thrombus, and arteriolar narrowing are seen, accompanied by lamina propria fibrosis crypt distortion. Endoscopic ablation therapies with laser are usually very effective for control of bleeding. ${ }^{[4]}$

Whereas for the urinary bladder, an early injury to the mucosa is mild, and epithelial and mucosal changes occur after 6 to 12 months, at the time of vascular changes. There will be submucosal telangiectasia along with thinning of the overlying epithelium. ${ }^{[3]}$ Imaging findings are more common in radiation cytopathic, as many of them are asymptomatic. Cystoscopy demonstrates erythema, edema, and neovascularisation. ${ }^{[5]}$

Stool softeners and antibiotics can manage mild rectal bleeding. For severe bleeding, endoscopic coagulation is the first line of management. ${ }^{[6]}$

Ureteral stricture and hemorrhagic cystitis are the two most common late manifestations of radiation. Hemorrhagic cystitis can be managed by laser fulguration of ectatic vessels. ${ }^{[6]}$

According to W.Majewski et al, the T stage of the tumor is the risk factor for bladder toxicity, and an increase in the radiation dose intensity is inversely proportional to the latency of bladder and bowel toxicity. ${ }^{[7]}$

Shinya Takemoto sample et al. have observed that steroid suppository or enema is the most effective pharmacotherapy for rectal bleeding secondary to chronic radiation proctitis and argon plasma coagulation is the most effective endoscopic intervention. ${ }^{[8]}$ 
We encountered two vesical bleed cases and one case of rectal bleed as a late complication of pelvic radiation. All three cases were females, who underwent radiation for carcinoma cervix, many years back. All three patients presented with severe bleeding and were taken up directly for endoscopic therapy and successfully managed.

\section{Conclusion}

Radiation therapy is the main component in the treatment of pelvic malignancies. It has early and late effects of toxicity on pelvic viscera, resulting in a variety of symptomatology. The radiologist should be well versed in post-radiation imaging appearances. CT angiogram should be the first choice of investigation in clinically stable patients presenting with hematuria and haematochezia. It picks up the site of active bleed and helps in targeted endoscopic or endovascular interventions.

\section{References}

1. Weintraub NL, Jones WK, Manka D. Understanding radiationinduced vascular disease. J Am Coll Cardiol. 2010;55(12):12371239. Available from: https://doi.org/10.1016/j.jacc.2009.11. 053.

2. Russell NS, Hoving S, Heineman S, Hage JJ, Woerdeman LAE, de Bree R, et al. Novel insights into pathological changes in muscular arteries of radiotherapy patients. Radiother Oncol. 2009;92(3):477-483. Available from: https://doi.org/10.1016/ j.radonc.2009.05.021.

3. Greven KM, Paunesku T. Radiation complications of the pelvis. Radiation toxicity: a practical guide. Boston, MA: Springer; 2008.

4. Kennedy G, Heise C. Radiation Colitis and Proctitis. Clin Colon Rectal Surg. 2007;20(1):64-72. Available from: https: //dx.doi.org/10.1055/s-2007-970202.

5. Maturen KE, Feng MU, Wasnik AP, Azar SF, Appelman HD, Francis IR, et al. Imaging Effects of Radiation Therapy in the Abdomen and Pelvis: Evaluating "Innocent Bystander" Tissues. Radio Graphics. 2013;33(2):599-619. Available from: https: //dx.doi.org/10.1148/rg.332125119.

6. Viswanathan AN, Lee LJ, Eswara JR, Horowitz NS, Konstantinopoulos PA, Mirabeau-Beale KL, et al. Complications of pelvic radiation in patients treated for gynecologic malignancies. Cancer. 2014;120(24):3870-3883. Available from: https://dx.doi.org/10.1002/cncr.28849.

7. Majewski W, Tarnawski R. Acute and Late Toxicity in Radical Radiotherapy for Bladder Cancer. Clin Oncol. 2009;21(8):598609. Available from: https://dx.doi.org/10.1016/j.clon.2009.04. 008.

8. Takemoto S, Shibamoto Y, Ayakawa S, Nagai A, Hayashi A, Ogino H. Treatment and prognosis of patients with late rectal bleeding after intensity-modulated radiation therapy for prostate cancer. Radiation Oncol. 2012;7:87-87. Available from: https: //dx.doi.org/10.1186/1748-717x-7-87.

Copyright: (C) the author(s), 2020. It is an open-access article distributed under the terms of the Creative Commons Attribution License (CC BY 4.0), which permits authors to retain ownership of the copyright for their content, and allow anyone to download, reuse, reprint, modify, distribute and/or copy the content as long as the original authors and source are cited.

How to cite this article: Pasupuleti J, Samireddypalle Y, Srujana G, Naik RR, Eada S, Vedaraju KS. Imaging Findings in LateOnset Rectal and Vesical Bleed in Patients Who Have Undergone Radiation Therapy for Pelvic Neoplasms . Asian J. Med. Radiol. Res. 2020;8(2):61-64.

DOI: dx.doi.org/10.47009/ajmrr.2020.8.2.10

Source of Support: Nil, Conflict of Interest: None declared. 